\title{
New CRLH-Based Planar Slotted Antennas with Helical Inductors for Wireless Communication Systems, RF-Circuits and Microwave Devices at UHF-SHF Bands
}

\author{
Mohammad Alibakhshi-Kenari ${ }^{1}$, Mohammad Naser-Moghadasi ${ }^{1}$, Ramazan Ali Sadeghzadeh ${ }^{2}$, Bal \\ Singh Virdee ${ }^{3}$ and Ernesto Limiti ${ }^{4}$ \\ ${ }^{1}$ Faculty of Eng., Science and Research Branch, Islamic Azad University, Tehran- IRAN \\ ${ }^{2}$ Faculty of Electrical and Computer Eng., K. N. University of Technology, Tehran- IRAN \\ ${ }^{3}$ London Metropolitan University, Center for Communications Technology, London N7 8DB, UK \\ ${ }^{4}$ Dipartimento di Ingegneria Elettronica, Università degli Studi di Roma Tor Vergata, Via del Politecnico 1, 00133 Roma - \\ ITALY \\ Corresponding author's email: *naeem.alibakhshi@yahoo.com
}

\begin{abstract}
Two novel planar slotted-antennas (PSAs) are presented that exhibit good radiation characteristics at the UHF-SHF bands. The proposed antennas are constructed using metamaterial unit-cells constituted from capacitive slots etched in the radiating patch and grounded spiral shaped inductive stubs. The proposed PSA design is fabricated on a commercially available dielectric substrate, i.e. Rogers RO4003 with permittivity of 3.38 and thickness of $1.6 \mathrm{~mm}$. The first PSA comprising five symmetrical unit-cells of slot-inductor-slot configuration operates over a wide bandwidth extending from $1-4.2 \mathrm{GHz}$ with a peak gain of $1.5 \mathrm{dBi}$ and efficiency of $35 \%$ at $2 \mathrm{GHz}$. The second PSA consists of ten asymmetrical unit-cells of slot-inductor configuration on the same size of substrate as the first PSA, enhances the antenna gain by $2 \mathrm{~dB}$ and efficiency by $25 \%$ and operates over $0.75-4.5 \mathrm{GHz}$. The asymmetrical unit-cell effectively increases the aperture size of the antenna without comprising its size. The electrical size of the antenna is $0.083 \lambda_{0} \times 0.033 \lambda_{0} \times 0.005 \lambda_{0}$, where freespace wavelength $\left(\lambda_{0}\right)$ is $1 \mathrm{GHz}$.
\end{abstract}

Keywords - Planar slotted antennas, metamaterial, composite right/left handed transmission-lines, UHF-SHF bands, RF circuits, microwave devices, wireless comunications systems.

\section{Introduction}

With the development of mobile communications, more and more communication standards with different working frequencies and protocols have been proposed and adopted. Antennas with wide-band/multi-band characteristics for various standards are therefore necessary. This has created a demand for broadband antennas that are low profile and occupy a small volume. Planar microstrip antennas are the most common form of printed antennas and these types of antennas have been used for many years since they have advantages of low-cost, conformability and ease of manufacturing though they have a narrow bandwidth. Among different planar antennas the slotted structure has the simplest structure, compact size and omnidirectional radiation pattern and is considered to be the best candidates for broadband applications [1]-[6].

This paper presents the results of investigation in developing compact planar slotted antenna (PSA) based on metamaterial (MTM) structures for broadband applications. The typical realization of MTM transmission-line (TL) is found in a quasi-lumped TL with elementary cells consisting of a series capacitor and a shunt inductor [7]. In practice, the losses in the MTM structure introduce series parasitic inductance in the capacitor and parasitic shunt capacitance in the inductor; thus the resulting MTM structure is essentially a composite right/lefthanded (CRLH) transmission-line structure [8][9].

In this paper the MTM is implemented on the patch antenna using a combination of capacitive slots etched in the patch, and grounded inductive spiral stubs. Ansoft's 3-D full-wave electromagnetic field software called High Frequency Structure Simulator (HFSS) [10] is used to model and analyze the proposed antenna. The initial antenna was optimized to improve its performance in terms of impedance bandwidth, gain and efficiency. This is achieved by simply increasing the number of inductive stubs without increasing the size of the original patch. The MTM structure essentially increases the aperture size of the antenna. The optimized antenna operates 
over $0.75-4.5 \mathrm{GHz}$ with a peak gain of $3.5 \mathrm{dBi}$ and efficiency of $60 \%$ at $2 \mathrm{GHz}$. The resulting PSA is compact and easy to fabricate.

\section{MTM-Based PSAs}

The main aims of the investigation were to realize a compact antenna with dimensions of $25 \times 10 \mathrm{~mm}^{2}$ and possessing good radiation characteristics in the UHF and SHF frequency bands. To achieve the aims the antenna design employs MTM-TL composed of series left-handed (LH) capacitors and shunt LH inductors. These reactive components were realized by etching capacitive slots on the patch and incorporating inductive spiral shaped stubs, which are terminated to the ground, on the patch. In practice, capacitive slot and inductive stub include parasitic right-handed ( $\mathrm{RH})$ effects, i.e. series $\mathrm{RH}$ inductance with the capacitive slot, and shunt $\mathrm{RH}$ capacitance associated with the inductive stub [11]. Hence, the proposed MTM antenna is more accurately described as a composite right/left-handed antenna.

The first antenna design includes a rectangular patch with five symmetrical unit-cells consisting of slotinductor-slot configuration. The slot is in the shape resembling a crotchet note "d", and the inductor is a spiral shaped stub that is grounded using a via-hole. This shape of the slot was determined through simulation investigation. It was found that variation in the slot capacitance contributed in improving the antenna's performance. Photograph of the fabricated PSA along with its equivalent circuit model are shown in Fig. 1. The antenna structure generates parasitic RH effect represented by the series inductance $\left(L_{R}\right)$ and the shunt capacitance $\left(C_{R}\right)$ resulting from current flowing over the antenna metallization that creates a voltage gradient between the metallization and the ground-plane. The model also includes RH lossy components $R_{R}$ and $G_{R}$, and LH lossy components $G_{L}$ and $R_{L}$, that account for the dielectric loss associated with $C_{L}$ and the ohmic loss associated with $L_{L}$. The PSA is constructed on Rogers RO4003 dielectric substrate with permittivity of 3.38, height from ground plane of $1.6 \mathrm{~mm}$ and loss tangent of 0.0022. The magnitudes of the parameters in Fig. 1 were determined from simulation analysis, and these are: $\mathrm{C}_{\mathrm{L}}=5.1 \mathrm{pF}, \mathrm{L}_{\mathrm{L}}=6.5 \mathrm{nH}, \mathrm{C}_{\mathrm{R}}=2.5 \mathrm{pF}, \mathrm{L}_{\mathrm{R}}=4.9 \mathrm{nH}, \mathrm{G}_{\mathrm{L}}=7 \mathrm{~S}$, $\mathrm{G}_{\mathrm{R}}=4.5 \mathrm{~S}, \mathrm{R}_{\mathrm{L}}=7.5 \Omega$, and $\mathrm{R}_{\mathrm{R}}=5.5 \Omega$.

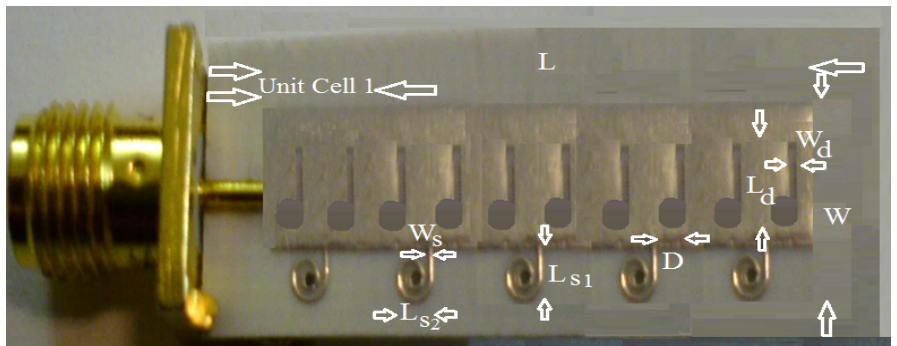

Fabricated prototype of Ant.\#1

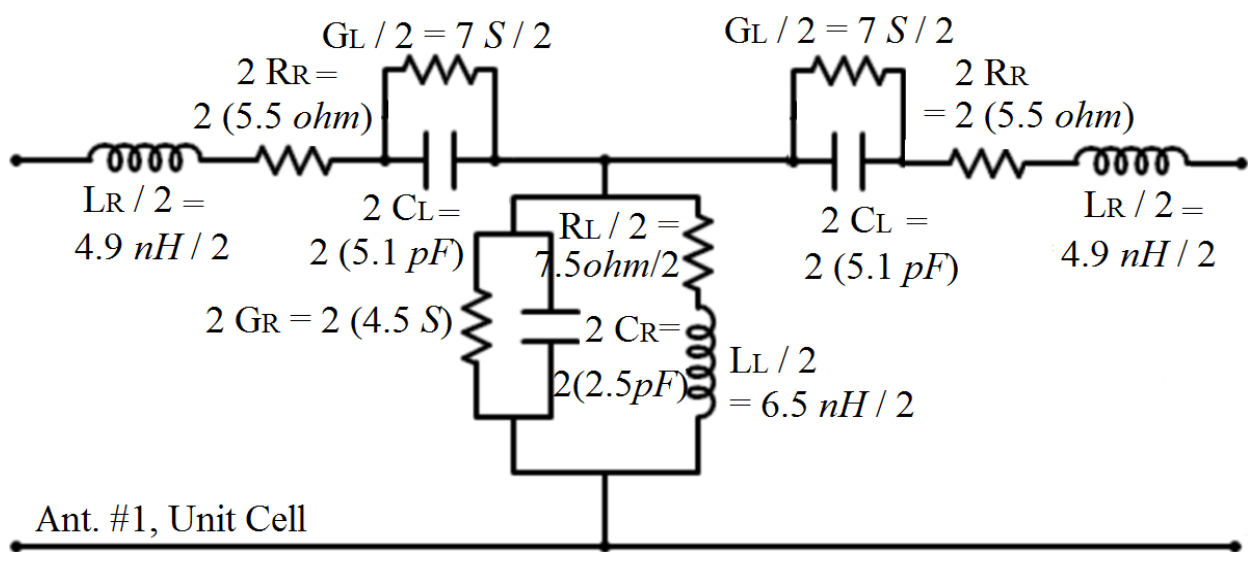

Equivalent circuit model of unit-cell for Ant.\#1

(a) Ant.\#1 with five symmetrical unit-cells. 


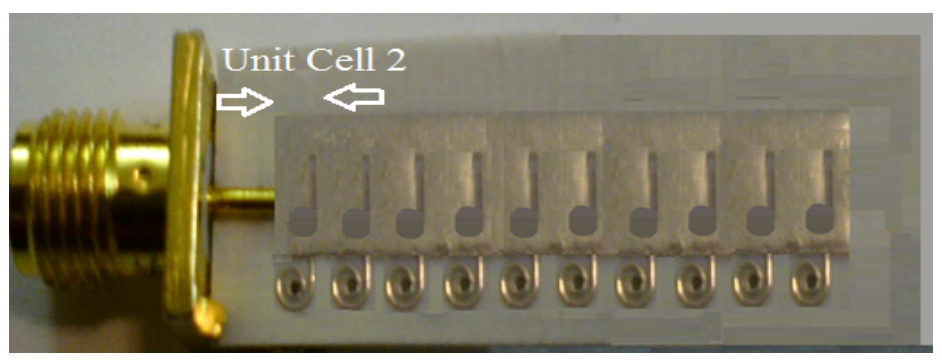

Fabricated prototype of Ant.\#2

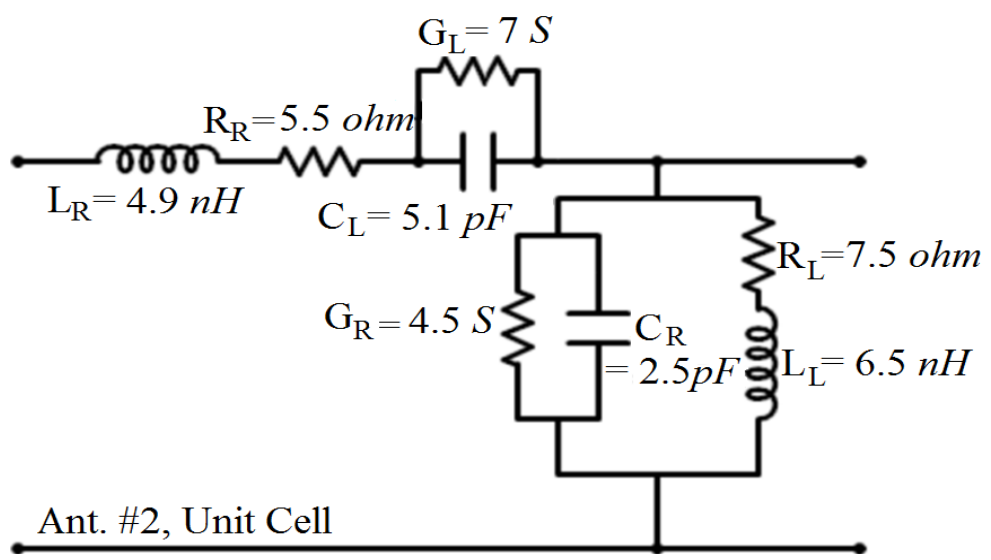

Equivalent circuit model of unit-cell of Ant.\#2

(b) Ant.\#2 with ten asymmetrical unit-cells. The size of this antenna is same as Ant.\#1

Fig. 1. Proposed two planar slotted antenna configurations.

The antenna is fed through a SMA connector which can cause an imbalance flow of current on the outer surface of the connector that can cause the connector to slightly radiate, which can cause a distortion in the radiation pattern. To negate this effect the second antenna is implemented using an asymmetrical unit-cell consisting of slot-inductor configuration, as shown in Fig.1 (b). The asymmetrical unit-cell consists of series LH capacitor with shunt LH inductance. The model also includes RH lossy components $R_{R}$ and $G_{R}$, and LH lossy components $G_{L}$ and $R_{L}$, that account for the dielectric loss associated with $C_{L}$ and the ohmic loss associated with $L_{L}$. The ten spiral inductors essentially concentrate the electromagnetic fields to be near antenna structure instead of spreading it over the antenna's ground-plane. This prevents unwanted coupling between the antenna and connector, and improves the radiation property of the antenna. The design parameters of proposed antenna along with its ground-plane size are listed in Table I. The dimensions of antenna and its ground-plane size are $25 \times 10 \times 1.6 \mathrm{~mm}^{3}$ and $28.5 \times 14.5 \mathrm{~mm}^{2}$, respectively.

TABLE I. DeSIGN PARAMETERS (units in mm, pF, $\mathrm{nH}, \mathrm{S}, \Omega$; unit-cell is abbreviated as UC)

\begin{tabular}{|c|c|c|c|c|c|c|c|c|c|}
\hline $\mathrm{UC} 1$ & $\mathrm{UC} 2$ & $L_{g}$ & $W_{g}$ & $L$ & $L_{d}$ & $L_{S 1}$ & $L_{S 2}$ & $W$ & $W_{d}$ \\
\hline 5 & 10 & 28.5 & 14.5 & 25 & 4 & 3 & 1.7 & 10 & 0.4 \\
\hline$W s$ & $D$ & $C_{L}$ & $L_{L}$ & $C_{R}$ & $L_{R}$ & $G_{L}$ & $G_{R}$ & $R_{L}$ & $R_{R}$ \\
\hline 0.2 & 1.4 & 5.1 & 6.5 & 2.5 & 4.9 & 7 & 4.5 & 7.5 & 5.5 \\
\hline
\end{tabular}

The simulated and measured reflection-coefficient response of the two antennas is shown in Fig. 2. Ant.\#2 with ten inductive stubs provides a wider bandwidth of $3.75 \mathrm{GHz}$ for $\mathrm{S}_{11}$ better than $-10 \mathrm{~dB}$ compared to Ant.\#1 which has a bandwidth of $3.2 \mathrm{GHz}$. Fig. 3 shows the gain and efficiency of both antennas. It shows Ant.\#1 has a peak gain and efficiency of $1.5 \mathrm{dBi}$ and $35 \%$ at $2 \mathrm{GHz}$, whereas Ant.\#2 has a peak gain and efficiency of $3.5 \mathrm{dBi}$ and $60 \%$ at $2 \mathrm{GHz}$. It is evident from Figs. 2 and 3, the gain and efficiency of Ant.\#2 is 
enhanced by $2 \mathrm{~dB}$ and $25 \%$, respectively, from Ant.\#1. In addition, the fractional bandwidth of Ant.\#2 increases by $19.78 \%$ from Ant.\#1. The results are given in Table II. These results indicate the effective aperture of the antenna is increased by doubling the inductive stubs from five to ten.

TABLE II. SUMMARY OF THE PROPOSED PSA PERFORMANCE

(Gain and efficiency are measured at 1, 2 and 4.2 GHz for Ant.\#1, and at 0.75, 2 and 4.5 GHz for Ant.\#2)

\begin{tabular}{|c|c|c|c|}
\hline & Ant.\#1 & Ant.\#2 & Change \\
\hline Dimensions & \multicolumn{2}{|c|}{$\begin{array}{c}25 \times 10 \times 1.6 \mathrm{~mm}^{3} \text { or } 0.083 \lambda_{0} \times 0.033 \lambda_{0} \times 0.005 \lambda_{0} @ 1 \mathrm{GHz} \\
\text { Ground-plane of Ants.: } 28.5 \times 14.5 \mathrm{~mm}^{2} \text { or } 0.098 \lambda_{0} \times 0.048 \lambda_{0}\end{array}$} & None \\
\hline \multirow[b]{2}{*}{ Bandwidth } & Sim.: $0.89-4.33 \mathrm{GHz} \approx 131.8 \%$ & Sim.: $0.58-4.7 \mathrm{GHz} \approx 156.06 \%$ & $24.26 \%$ \\
\hline & Meas.: $1-4.2 \mathrm{GHz} \approx 123.07 \%$ & $\begin{array}{l}\text { Meas.: } 0.75-4.5 \mathrm{GHz} \\
\quad \approx 142.85 \%\end{array}$ & $19.78 \%$ \\
\hline Gain & $0.2,1.5$ and $1 \mathrm{dBi}$ & $0.25,3.5$ and $2.7 \mathrm{dBi}$ & $2 \mathrm{~dB}$ increase @ $2 \mathrm{GHz}$ \\
\hline Efficiency & 11,35 and $27 \%$ & 13,60 and $48 \%$ & $25 \%$ increase @ $2 \mathrm{GHz}$ \\
\hline
\end{tabular}

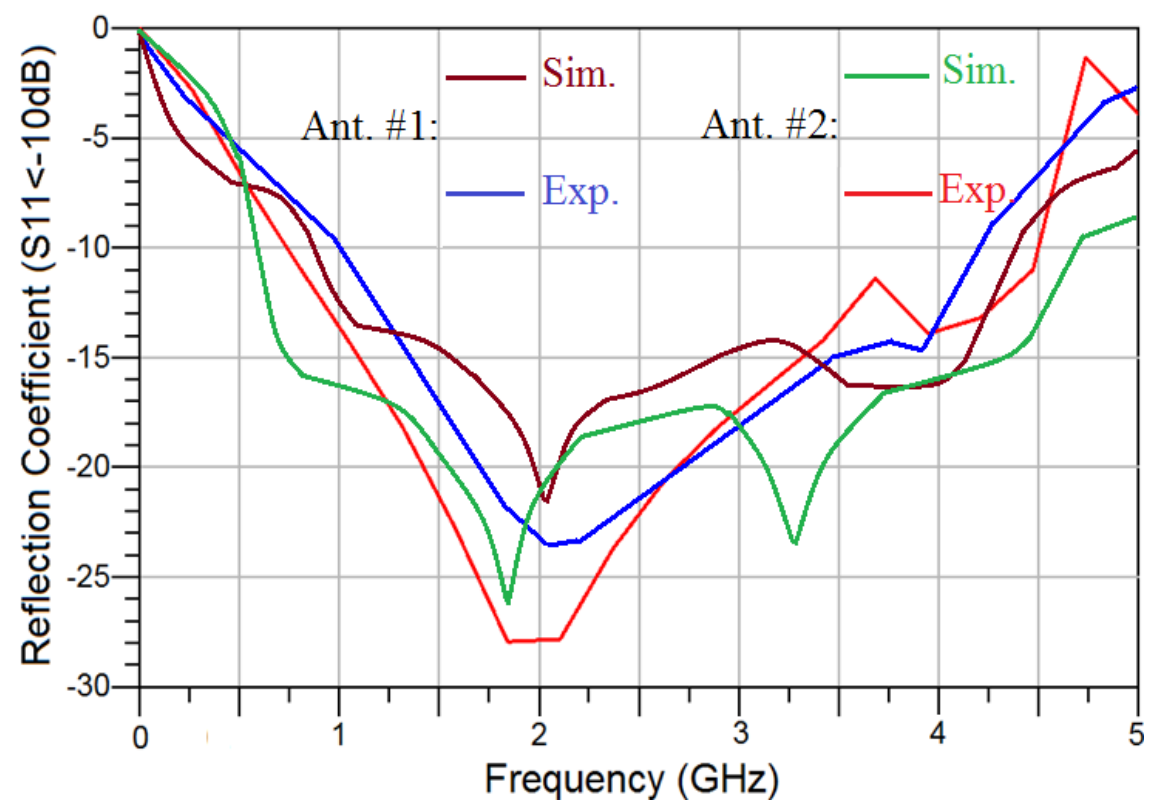

Fig. 2. Simulated and measured reflection-coefficient responses of the two proposed antennas. Measured impedance bandwidth improves from $123.07 \%$ (for Ant.\#1) to $142.85 \%$ (for Ant.\#2). 


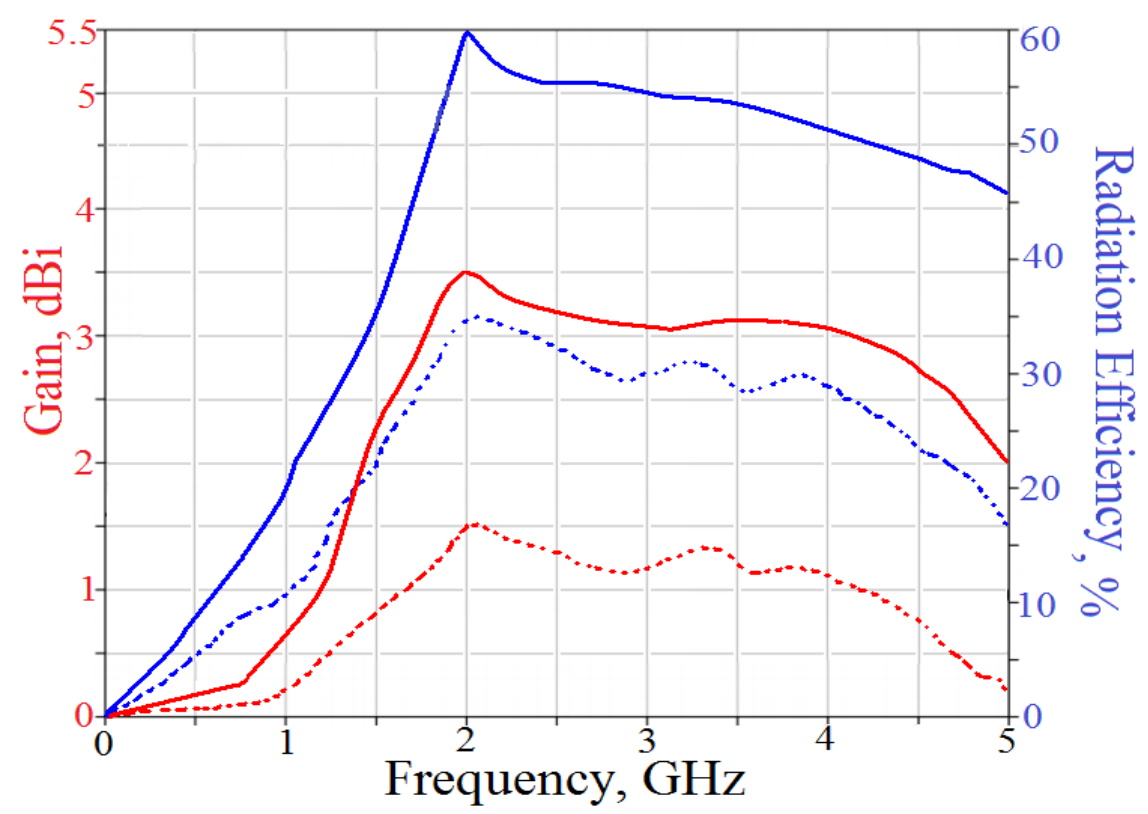

Fig. 3. Measured gain and efficiency performance as a function of frequency for the two proposed antennas. (Ant.\#1: Dashed-lines, and Ant.\#2: Solid-lines).

The measured E-plane and H-plane radiation patterns of both antennas are shown in Fig. 4. Ant.\#1 has Eand $\mathrm{H}$-plane radiation patterns similar to a dipole antenna with a maximum gain at 180 degrees azimuth at $1 \mathrm{GHz}$ however the direction of the maxima gain flips to around 0 degrees at $2 \mathrm{GHz}$. At $4 \mathrm{GHz}$ the maximum flips back to at 180 degrees. This indicates the phase of radiation from each unit-cell changes with frequency. The radiation pattern of Ant.\#1 is predominately bidirectional. Ant.\#2 has bidirectional E- and H-plane radiation patterns at 1 $\mathrm{GHz}$ which becomes omnidirectional at $2 \mathrm{GHz}$. This shows that the additional inductive lines suppress the effects of imbalance flow of current on the outer conductor of the SMA connector and enhance its impedance bandwidth. The gain of the H-plane radiation is reduced by about $3 \mathrm{~dB}$ at $2 \mathrm{GHz}$ around 90 and 270 degrees. At $4 \mathrm{GHz}$ the E-plane radiation has a null at 180 degree.
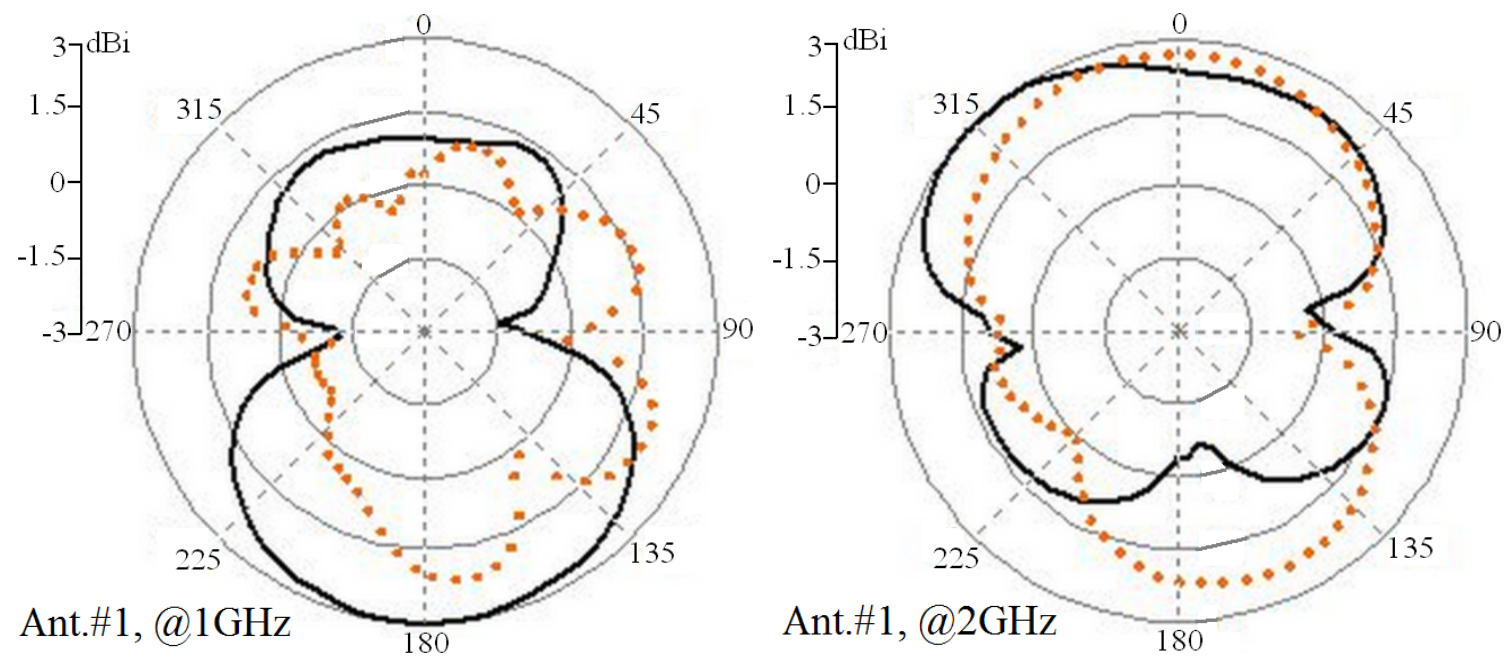

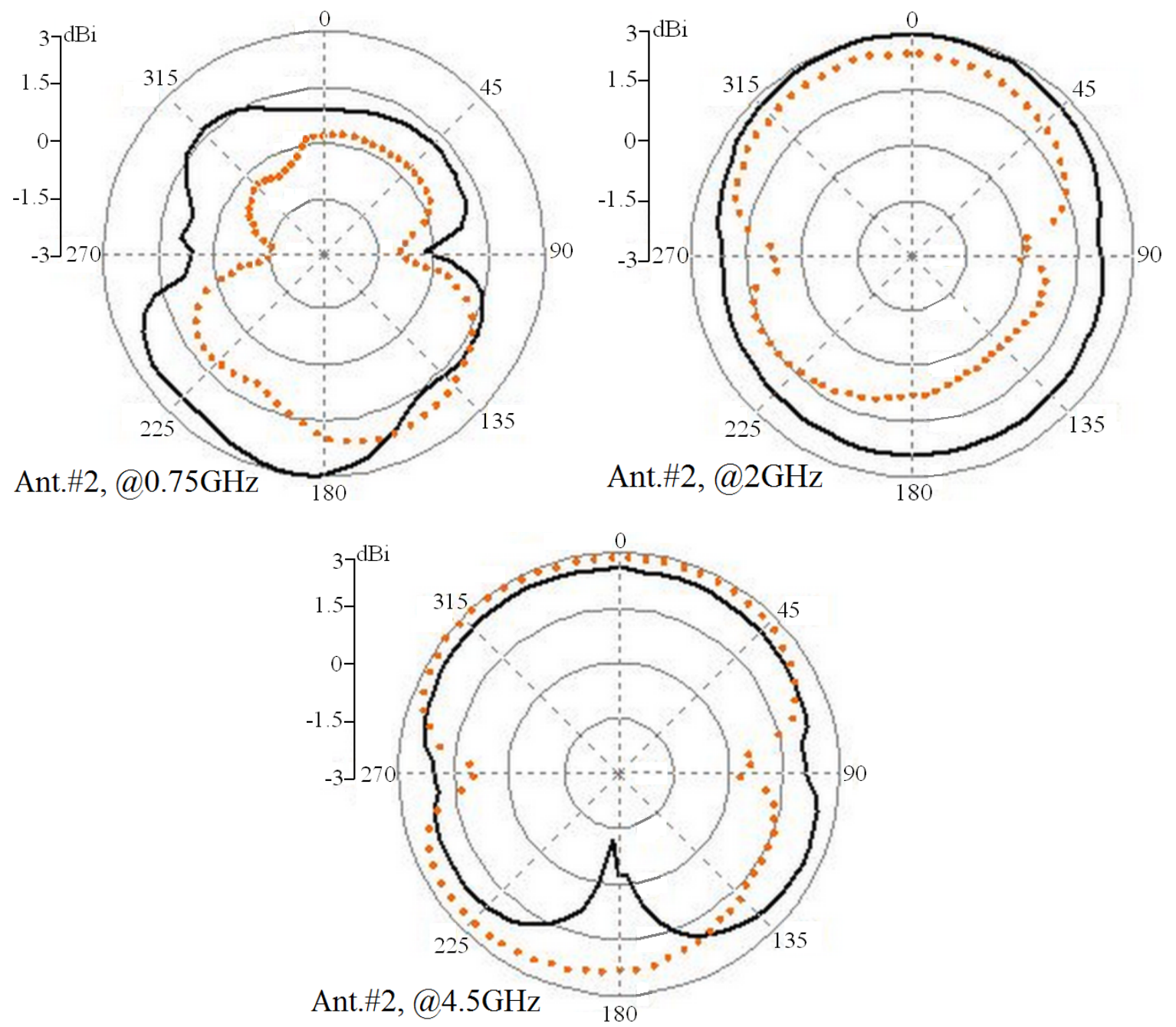

Fig. 4. Measured E-plane and H-plane radiation patterns at the several operating frequencies.

(Black solid-line and color dashed-line represent E- and H-planes, respectively).

The measured performance of the prototyped antenna in Fig.2 does not accurately agree with the simulated performance. This discrepancy is prominent at lower frequencies and is due to the feed cable and unwanted coupling with the SMA connector. In the simulation the antenna is fed directly with a signal source without using a feeding cable. However, in practice the measurement of the prototype antenna was measured with Agilent's 8722ES vector network analyzer connected to the antenna using a coaxial cable. Also, the small ground-plane of the antenna cannot approximate an infinite ground-plane, which causes surface current flow over the connector and cable due to unwanted field coupling between the antenna and the connector. The fabricated antenna has benefits of simple layout which is easy to fabricate at low cost, is low profile and easy to integrate with RF circuits, and has overall characteristics for use in the broadband wireless communication.

\section{Conclusions}

A compact and low profile antenna based on metamaterial unit-cells is shown to provide broadband performance for wireless system operating over UHF-SHF bands. It is shown the implementation of the unit-cells comprising a slot in a shape of a crotchet note and grounded spiral stub provides improvement in gain and efficiency of 3.5 $\mathrm{dBi}$ and $60 \%$, respectively, at $2 \mathrm{GHz}$, as well as impedance bandwidth of $142.85 \%$ and excellent radiation characteristics. The antenna was fabricated on Rogers RO4003 dielectric substrate has dimensions of $25 \times 10 \times 1.6$ $\mathrm{mm}^{3}$. 


\section{References}

[1] Liu L., Cheung S.W., Yuk T.I., "Bandwidth Improvements Using Ground Slots for Compact UWB Microstrip-fed Antennas,” Progress in Electromagnetics Research Symposium (PIERS), 2011. Suzhou, China.

[2] Cheung S.W., Liu L., Azim R., Islam M.T., "A Compact Circular-Ring Antenna for Ultra-Wideband Applications," Microwave and Optical Technology Letters, Vol.53, 2011, pp. 2283-2288.

[3] Sun Y.Y., Cheung S.W., Yuk T.I., "Studies of Planar Antennas with Different Radiator Shapes for Ultra-wideband Body-centric Wireless Communications," Progress in Electromagnetics Research Symposium (PIERS), 2011. Suzhou, China. [4] Zhang J., Sun X.L., Cheung S.W., Yuk T.I., "CPW-Coupled-Fed Elliptical Monopole Antenna for UWB Applications," IEEE Radio Wireless Week, Jan 2012, pp, 295-298.

[5] Sun Y.Y., Islam M.T., Cheung S.W., Yuk T.I., Azim T., Misran N., “Offset-fed UWB Antenna with Multi-slotted Ground Plane,” IEEE International Workshop on Antenna Technology, 2011, pp. 432-436.

[6] Sun Y.Y., Cheung S.W., Yuk T.I., "Planar Monopole Ultra-wideband Antennas with Different Radiator Shapes for Bodycentric Wireless Networks," Progress in Electromagnetics Research Symposium, 2012, Kuala Lumpur, Malaysia.

[7] Engheta N., Ziolkowski, R.W., "Electromagnetic Metamaterials: Physics and Engineering Explorations," Wiley and IEEE Press, Hoboken, 2006.

[8] Alibakhshi-Kenari M., Naser-Moghadasi M., Virdee B.S., Andujar A., Anguera, J., "Compact Antenna based on a Composite Right/Left Handed Transmission Line,” Microwave and Optical Technology Letters, Vol. 57, Issue 8, 2015 , pp. $1785-1788$.

[9] Eleftheriades, G.V., "EM Transmission-line Metamaterials,” Vol. 12, No. 3, Mar. 2009, pp. 30-41.

[10] Ansoft HFSS, www.ansoft.com/products/hf/hfss.

[11] Lai A., Caloz C., and Itoh T., "Composite Right/Left Handed Transmission Line Metamaterials," IEEE Microwave Mag., Vol. 5, No. 3, Sept. 2004, pp. 34-50. 